OPEN ACCESS

Edited by:

David William Waite,

The University of Auckland,

New Zealand

Reviewed by:

Brittany Leigh,

Vanderbilt University, United States

Steve Perlman,

University of Victoria, Canada

*Correspondence:

Sulochana K. Wasala

wasalaw@oregonstate.edu

Specialty section:

This article was submitted to

Microbial Symbioses,

a section of the journal

Frontiers in Microbiology

Received: 18 January 2019 Accepted: 16 April 2019

Published: 07 May 2019

Citation:

Wasala SK, Brown AMV, Kang J, Howe DK, Peetz AB, Zasada IA and Denver DR (2019) Variable

Abundance and Distribution of Wolbachia and Cardinium Endosymbionts in Plant-Parasitic

Nematode Field Populations.

Front. Microbiol. 10:964.

doi: 10.3389/fmich.2019.00964

\section{Variable Abundance and Distribution of Wolbachia and Cardinium Endosymbionts in Plant-Parasitic Nematode Field Populations}

\author{
Sulochana K. Wasala ${ }^{1 *}$, Amanda M. V. Brown'2, Jiwon Kang', Dana K. Howe', \\ Amy B. Peetz ${ }^{3}$, Inga A. Zasada ${ }^{3}$ and Dee R. Denver ${ }^{1}$
}

1 Department of Integrative Biology, Oregon State University, Corvallis, OR, United States, ${ }^{2}$ Department of Biological Sciences, Texas Tech University, Lubbock, TX, United States, ${ }^{3}$ USDA-ARS Horticultural Crops Research Laboratory, Corvallis, OR, United States

The bacterial endosymbiont Wolbachia interacts with different invertebrate hosts, engaging in diverse symbiotic relationships. Wolbachia is often a reproductive parasite in arthropods, but an obligate mutualist in filarial nematodes. Wolbachia was recently discovered in plant-parasitic nematodes, and, is thus far known in just two genera Pratylenchus and Radopholus, yet the symbiont's function remains unknown. The occurrence of Wolbachia in these economically important plant pests offers an unexplored biocontrol strategy. However, development of Wolbachia-based biocontrol requires an improved understanding of symbiont-host functional interactions and the symbiont's prevalence among nematode field populations. This study used a moleculargenetic approach to assess the prevalence of a Wolbachia lineage (wPpe) in 32 field populations of Pratylenchus penetrans. Populations were examined from eight different plant species in Washington, Oregon, and California. Nematodes were also screened for the endosymbiotic bacterium Cardinium (cPpe) that was recently shown to co-infect $P$. penetrans. Results identified wPpe in 9/32 and cPpe in 1/32 of $P$. penetrans field populations analyzed. No co-infection was observed in field populations. Wolbachia was detected in nematodes from 4/8 plant-hosts examined (raspberry, strawberry, clover, and lily), and in all three states surveyed. Cardinium was detected in nematodes from mint in Washington. In the wPpe-infected $P$. penetrans populations collected from raspberry, the prevalence of wPpe infection ranged from 11 to $58 \%$. This pattern is unlike that in filarial nematodes where Wolbachia is an obligate mutualist and occurs in $100 \%$ of the host. Further analysis of wPpe-infected populations revealed femaleskewed sex ratios (up to 96\%), with the degree of skew positively correlating with wPpe prevalence. Uninfected nematode populations had approximately equal numbers of males and females. Comparisons of 54 wPpe $16 S$ ribosomal RNA sequences revealed high similarity across the geographic isolates, with 45 of 54 isolates being identical at this locus. The complete absence of wPpe among some populations and low prevalence 
in others suggest that this endosymbiont is not an obligate mutualist of $P$. penetrans. The observed sex ratio bias in wPpe-infected nematode populations is similar to that observed in arthropods where Wolbachia acts as a reproductive manipulator, raising the question of a similar role in plant-parasitic nematodes.

Keywords: bacterial endosymbionts, nematode, sex ratio distortion, Wolbachia, Cardinium, Pratylenchus penetrans

\section{INTRODUCTION}

Microbial symbiosis is increasingly recognized as an integral component of ecosystems with a major role in ecology and evolution. These symbiotic relationships range from mutualism (where both symbiotic partners benefit) to commensalism (where one symbiont benefits, while the other has no notable fitness effect) to parasitism (where one symbiont is harmed). Symbiotic relationships between microbes and multicellular hosts are often subdivided into ectosymbiosis such as gammaproteobacteria associated with the external surfaces of marine invertebrates (Goffredi, 2010), and endosymbiosis such as photosynthetic Symbiodinium within cnidarian cells and alphaproteobacteria within insect cells (Werren et al., 2008; Fransolet et al., 2012). Endosymbiosis involves intimate interactions between organisms, in which a microbial partner lives within its host cells. Many microbial symbioses likely originated as facultative relationships, which over time evolved into obligate relationships (Moya et al., 2008; Lamelas et al., 2011; Zug and Hammerstein, 2014). Further, eukaryotic organelles such as mitochondria and chloroplasts evolved from endosymbiotic events involving alphaproteobacterial and cyanobacterial ancestors, respectively. Microbial symbionts also serve as an exciting potential target for disease management and prevention. Despite the basic and applied importance of microbial symbiosis, ecological, and functional details remain to be understood in many systems.

Wolbachia is a common bacterial endosymbiont occurring in arthropods and nematodes. It was first detected in the mosquito Culex pipiens (Hertig, 1936) and since then has become established as a successful biocontrol agent for many pests and disease vectors (Brelsfoard and Dobson, 2009; Slatko et al., 2014). Wolbachia has varying interaction with invertebrate hosts, ranging from parasitic to mutualistic. In arthropods, Wolbachia typically acts as a reproductive parasite inducing a range of reproductive perturbations. Wolbachia induced reproductive abnormalities in arthropod hosts are often described in four categories: cytoplasmic incompatibility (CI), male-killing, genetic male feminization, and parthenogenesis-induction (Werren et al., 2008). Wolbachia-induced parthenogenesis is often observed in species with haplodiploid sex determination, in which males develop from unfertilized eggs and are haploid (Werren et al., 2008). Here, Wolbachia induces diploid development of unfertilized eggs so that infected females produce daughters instead of sons. Parthenogenesis-inducing Wolbachia phenotypes have been observed in Trichogramma wasps, mites, and thrips (Stouthamer et al., 1990; Arakaki et al., 2001; Weeks and Breeuwer, 2001). Wolbachia-induced feminization results in genetic males developing into functional females, as has been described in isopods, and insects in the orders Lepidoptera, and Hemiptera (Kageyama et al., 2002; Vandekerckhove et al., 2003; Negri et al., 2006; Kern et al., 2015). Wolbachia is also reported to selectively kill male hosts, mainly during embryogenesis (Werren et al., 2008), in the arthropod orders Coleoptera, Diptera, Lepidoptera, and in Pseudoscorpiones (Fialho and Stevens, 2000; Hurst et al., 2000; Jiggins et al., 2000; Dyson and Hurst, 2004; Zeh et al., 2005). Wolbachia primarily undergoes vertical transmission, through host eggs and not via sperm (Werren et al., 2008). Thus, the evolutionary advantages of these symbionts often involve improving transmission by increasing the proportion of females in the host population. Consequently, except for $\mathrm{CI}$, the reproductive abnormalities induced by Wolbachia distort sex-ratio of the host population toward females (Cordaux et al., 2011).

$\mathrm{CI}$ is the most frequently observed Wolbachia phenotype in which sperm from Wolbachia-infected males are incompatible with eggs from females that do not harbor Wolbachia (or the same Wolbachia strain), such that resulting crosses result in no viable offspring. This phenomenon was first observed in the mosquito C. pipiens (Hertig, 1936), and has since been described in other insect orders, arachnids, and isopods (Bourtzis et al., 1996; Sicard et al., 2014; Xie et al., 2016). As a result of CI, Wolbachia in arthropods can cause a form of conditional sterility that can be used to suppress populations of medically and economically important insects (Slatko et al., 2014). For example, introduction of Wolbachia into medfly Ceratitis capitate, and disease-causing mosquito populations was shown to suppress those insect pest populations (Zabalou et al., 2004; Flores and O’Neill, 2018). By contrast, in animal and human-parasitic filarial nematodes, Wolbachia acts as an obligate mutualist and is required for normal host development, fertility, and survival. Treating filarial nematode-infected animals and humans with antibiotics that eliminate Wolbachia resulted in the prevention of nematode embryo development (Bandi et al., 1999), growth retardation/infertility (Hoerauf et al., 1999), and eventual death (Langworthy et al., 2000). Since treatments with antibiotics that eliminate mutualistic Wolbachia in filarial nematodes have adverse effects on the symbiont host, this approach has been used for the treatment of human filariasis (Taylor et al., 2005; Turner et al., 2017).

Previously, there was only one reported case of Wolbachia infecting a plant-parasitic nematode genus: Radopholus (burrowing nematode) (Haegeman et al., 2009). We recently discovered Wolbachia in another plant-parasitic nematode, Pratylenchus penetrans (root-lesion nematode), through genome skimming and fluorescence in situ hybridization (FISH) analyses (Brown et al., 2016; Denver et al., 2016). This Wolbachia 
strain was designated wPpe. P. penetrans is an amphimictic nematode species, with males and females reported as occurring in approximately equal numbers (Roman and Triantaphyllou, 1969), although there was one previously reported case of P. penetrans populations being female-biased (Tarte and Mai, 1976). The $P$. penetrans population in which wPpe was initially discovered, also carried another endosymbiotic bacterium Cardinium (cPpe) (Brown et al., 2016; Denver et al., 2016). Cardinium is often found with Wolbachia in arthropods but has not been reported to occur in filarial nematodes. However, Cardinium has been reported in two other plantparasitic nematode genera; Heterodera (soybean-cyst nematode) (Shepherd et al., 1973; Endo, 1979; Noel and Atibalentja, 2006; Brown, 2018) and Globodera (potato-cyst nematode) (Walsh et al., 1983a,b; Brown, 2018).

Bacterial symbioses in plant-parasitic nematodes have generally received limited research attention, except for the bacterial genus Pasteuria (Atibalentja and Noel, 2008). However, plant-parasitic nematodes are responsible for more than US \$100 billion in annual agriculture loss worldwide (Bird and Kaloshian, 2003) and managing these parasites through the manipulation of their microbial symbionts offers an appealing approach. The genus Pratylenchus is among the top three most significant plant-parasitic nematodes (Jones et al., 2013), infecting many important crop plants. The conventional methods of controlling this nematode such as crop rotation are generally not successful because of its wide host range. Further, resistant plants are limited to a few crops. Fumigant and non-fumigant nematicides are used to control this nematode, however, there are concerns with these control strategies because of environmental and health-related issues. The discovery of wPpe in this species motivates further exploration of host-endosymbiont interactions in this system centered on its potential as a biological control agent against $P$. penetrans. Furthermore, wPpe is intriguing from a basic biological viewpoint, because of its place as the earliest-branching Wolbachia lineage known to date, diverging prior to the Wolbachia clades infecting arthropods and filarial nematodes (Brown et al., 2016).

The first description of wPpe was based on a greenhouse $P$. penetrans population, leaving open questions about its prevalence and diversity in field populations, and its potential phenotypic effects on its host. The present study addresses these important gaps in Wolbachia symbiosis knowledge using multiple $P$. penetrans field populations in the Northwestern United States. Our specific objectives in this study were to (a) investigate the prevalence of wPpe in $P$. penetrans for a wide range of field populations using a molecular genetic approach, and (b) explore the potential for wPpe to impact host reproduction, which together serve to advance our hypotheses about the possible symbiotic role for this Wolbachia strain.

\section{MATERIALS AND METHODS}

\section{Nematode Populations and Collection}

We collected $P$. penetrans populations from 32 commercial crop fields occurring in 11 different locations in three states
(Washington, Oregon, and California) along the West Coast of the United States for this study (Figure 1). The nematode populations were obtained from eight different host plants (Table 1). Additionally, a population of $P$. penetrans maintained in the greenhouse at the USDA-ARS Horticultural Research Crops Laboratory (Corvallis, Oregon) from which wPpe was originally discovered (Brown et al., 2016) was considered. The greenhouse population is maintained on raspberry and was originally collected from raspberry fields in Oregon and Washington. To obtain $P$. penetrans from the fields, multiple root and soil samples were collected from random areas in a field and then combined into a single composite sample. Nematodes were extracted from roots by intermittent mist (Gigot et al., 2013). Root samples were washed free of soil, cut into small pieces $(<5 \mathrm{~cm})$ and placed on screens over funnels draining into test tubes and misted at frequent intervals for 5 days. Nematodes were extracted from soil by placing $50 \mathrm{~g}$ on a Baermann funnel with nematodes collected after 5 days. $P$. penetrans were collected in water and stored at $4^{\circ} \mathrm{C}$ until analyzed.

$P$. penetrans field populations collected for this study $(N=32)$ were used to assess the occurrence of endosymbionts (Wolbachia wPpe and Cardinium cPpe). Depending on the availability, all populations obtained from raspberry $(N=10)$ were used for within-population wPpe prevalence assessment, wPpe 16S

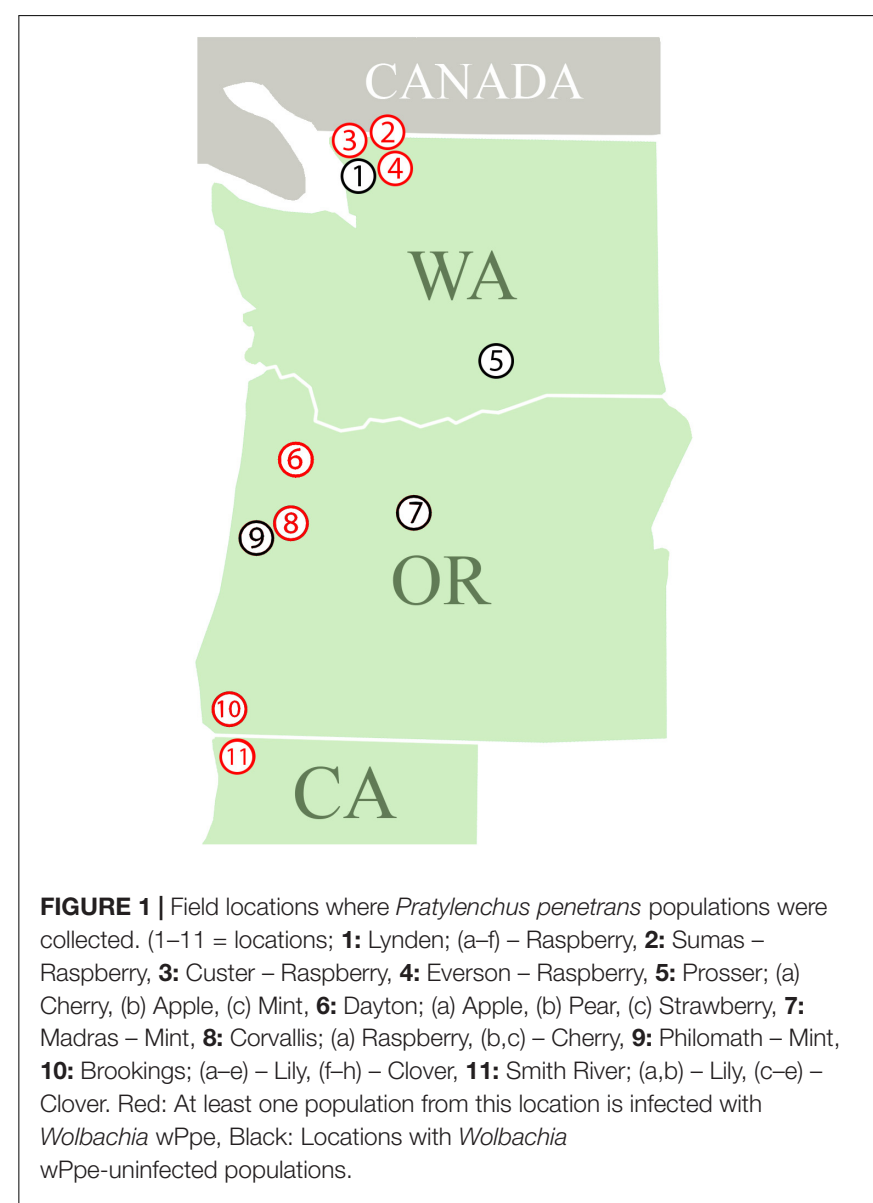


TABLE 1 | Occurrence of Wolbachia wPpe and Cardinium cPpe in Pratylenchus penetrans populations.

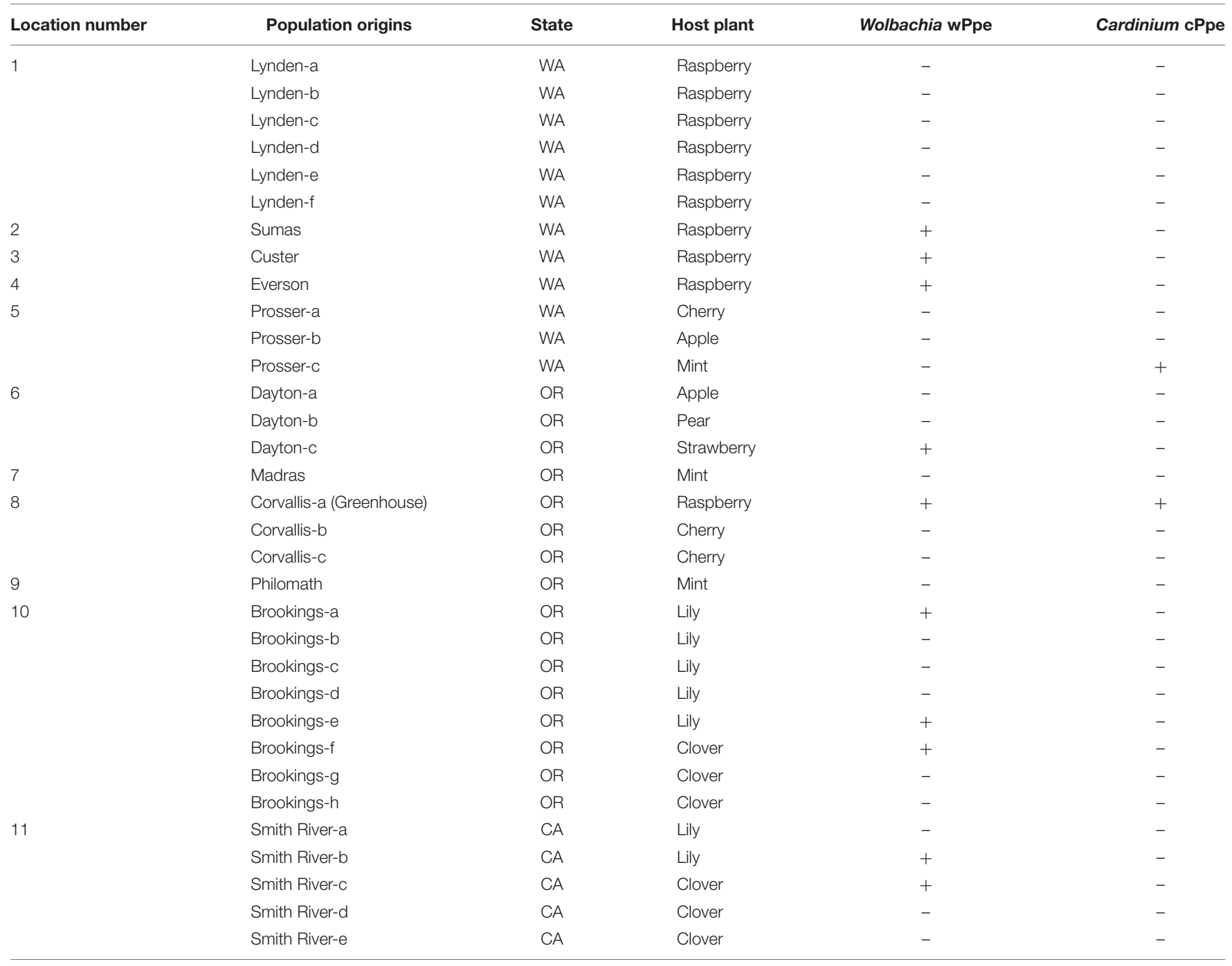

"+" indicates endosymbiont presence based on PCR, "-" indicates endosymbiont absence based on PCR. DNA was extracted from 200 nematodes for bulk PCR.

ribosomal RNA (rRNA) sequence analysis and $P$. penetrans sex ratio determination.

\section{Molecular-Genetic Detection of Endosymbionts in $P$. penetrans}

Nematodes $(N=7,183$ total $)$ were screened for the presence of wPpe and cPpe using PCR-based strategies. Two types of DNA samples were screened: (1) DNA prepared from many nematodes originating from the same population ("bulk" samples), (2) DNA prepared from individual nematodes (performed for $P$. penetrans populations obtained from raspberry based on the infection status).

\section{DNA Extraction}

For bulk extractions, $P$. penetrans $(N=200)$ from each population were prepared for DNA isolation in $75 \mu$ l of Worm Lysis Buffer (WLB): $1 \mathrm{ml}=880 \mu \mathrm{l}$ of $\mathrm{dH}_{2} \mathrm{O}, 50 \mu \mathrm{l}$ of $50 \mathrm{mM} \mathrm{KCl}, 50 \mu \mathrm{l}$ of $0.05 \%$ gelatin, $4.5 \mu \mathrm{l}$ of $0.45 \%$ Tween $20,10 \mu \mathrm{l}$ of $10 \mathrm{mM}$ Tris, $3.3 \mu \mathrm{l}$ of $60 \mu \mathrm{g} / \mathrm{ml}$ Proteinase $\mathrm{K}$ and $2.5 \mu \mathrm{l}$ of $2.5 \mathrm{mM} \mathrm{MgCl}_{2}$.
Samples were freeze-thawed five times to break the nematode cuticle (frozen at $-80^{\circ} \mathrm{C}$ for $>10 \mathrm{~min}$ and thawed at room temperature for $5 \mathrm{~min}$ ) before being digested at $60^{\circ} \mathrm{C}$ for $90 \mathrm{~min}$, followed by a $95^{\circ} \mathrm{C}$ incubation for $15 \mathrm{~min}$ for DNA extraction. For single-nematode DNA extractions, individual nematodes were picked into $15 \mu \mathrm{l}$ of WLB, and then followed the same treatment as bulk samples.

\section{Primer Design, PCR Amplification, and DNA Sequencing}

A more sensitive nested PCR which is generally required for Wolbachia surveys (Wolfgang et al., 2009) was performed using newly designed wPpe-specific 16S rRNA primers (Supplementary Table S1). Our nested PCR strategy was designed to minimize non-specific primer binding. The first set of primers were designed by aligning Wolbachia 16S rRNA sequences from $P$. penetrans and $R$. similis in MEGA6 (Kumar et al., 2008) to amplify a $545 \mathrm{bp}$ fragment. While this primer 
set matches the 16S rRNA sequence from $P$. penetrans and $R$. similis Wolbachia strains, it also targets other Wolbachia strains and a few other proteobacteria. The second primer set specifically targets the 16S rRNA sequence of Wolbachia strains from only $P$. penetrans and $R$. similis, and includes several mismatches to sequences from other bacteria, including sister alphaproteobacteria. The second set of primers amplifies a 382 bp fragment of the 16S rRNA gene of Wolbachia (primer locations relative to E. coli $16 \mathrm{~S}$ rRNA shown in Supplementary Figure S1). Primers were tested with $P$. penetrans obtained from the greenhouse population where Wolbachia was first detected and confirmed by agarose gel electrophoresis and DNA sequencing. Caenorhabditis elegans, a nematode known to not harbor Wolbachia, was used as a negative control to check for any non-specific binding of the primers.

Nested PCR was started with $1 \mu$ l of genomic DNA from bulk DNA extractions or $3 \mu \mathrm{l}$ of genomic DNA from single nematode DNA extractions. PCR reactions, $50 \mu \mathrm{l}$, were performed for Wolbachia screens: $5 \mu \mathrm{l}$ of $10 \times$ Taq buffer, $1 \mu \mathrm{l}$ of $10 \mu \mathrm{M}$ dNTPs, $2 \mu \mathrm{l}$ of $10 \mu \mathrm{M}$ forward primer, $2 \mu \mathrm{l}$ of $10 \mu \mathrm{M}$ reverse primer, $0.3 \mu \mathrm{l}$ of Taq polymerase, 1 or $3 \mu \mathrm{l}$ of genomic DNA, 38.7 or $36.7 \mu \mathrm{l}$ of nuclease-free water. The products from the first PCR reaction were diluted 1:10 and used as a template for the second, more specific PCR. To avoid false positives due to contamination, negative controls (sterile water instead of sample DNA) were analyzed for each PCR. All PCR products were visualized on 1.5\% agarose gels stained with ethidium bromide.

For wPpe-infected raspberry $P$. penetrans populations (previously identified by bulk DNA PCRs), individual nematode PCR was performed to determine the prevalence of infected nematodes in the source population. Adult females and males were visually identified based on their morphological characters for individual nematode PCR. Individual females $(N=100)$ and males $(N=24-100)$ from each population were analyzed by nested PCR as described above. DNA sequencing of PCR products was performed at the Center for Genome Research and Biocomputing (Oregon State University) to confirm amplification of the correct target.

PCR was performed for bulk DNA extractions using Cardinium-specific 16S rRNA primers to screen for cPpe (Brown et al., 2018) followed by DNA sequencing to confirm amplification of the correct target. For the bulk nematode samples shown to be uninfected (by either wPpe or cPpe), three PCR replicates were performed using newly extracted bulk nematode DNA to avoid possible false negatives. For bulk and individual nematode samples, PCR was also performed using $P$. penetrans-specific mitochondrial DNA (mtDNA) primers (Supplementary Table S2), to test for possible false negatives due to inhibitors or improper extraction or digestion of nematode DNA. $P$. penetrans mtDNA primers were designed so that the forward primer targeted $t R N A^{A s p}$ and the reverse primer targeted coxl gene, resulting in an amplicon of $\sim 840 \mathrm{bp}$. Samples that did not amplify with mtDNA primers were discarded and not counted in this study. After the gel electrophoresis, the mtDNA PCR products were sequenced and amplification of the correct target was confirmed by blastn searches against NCBI databases.

\section{wPpe Prevalence Calculation}

After the percentages of infected males and females were calculated, total wPpe prevalence in a nematode population was calculated as described below. When the nematode sex ratio is 1:1, the Wolbachia percentage was calculated as:

$$
\begin{aligned}
& \text { Wolbachia } \%= \\
& \frac{\text { (number of males infected }+ \text { number of females infected) }}{\text { Total number of individuals }}
\end{aligned}
$$

However, when the nematode sex ratio is not $1: 1$, the male and female nematode percentage in the population were taken into account to estimate the Wolbachia percentage. Therefore, the Wolbachia percentage was calculated as below.

Wolbachia $\%=$

$\frac{(\% \text { males } \times \% \text { infected males })+(\% \text { females } \times \% \text { infected females })}{100}$

For 1:1 sex ratio, the Equation (2) can be written as below, which is another representation of Equation (1).

$$
\begin{aligned}
& \text { Wolbachia } \%= \\
& \frac{(50 \times \% \text { infected males })+(50 \times \% \text { infected females })}{100}
\end{aligned}
$$

\section{Wolbachia 16S rRNA Sequence Analysis and Phylogenetics}

Multiple alignments and phylogenetic analyses of 16S rRNA sequences were performed using MEGA6 (Kumar et al., 2008). DNA sequence multiple alignments were performed using the ClustalW function in MEGA6; the IUB DNA weight matrix was used and the gap-opening and extension penalties were set to 15 and 6.66, respectively (default settings). Reliabilities of resultant multiple alignments were evaluated by visual inspection after running ClustalW.

To evaluate the $16 \mathrm{~S}$ rRNA diversity of wPpe lineages in different $P$. penetrans populations, we sequenced 54 wPpe 16S rRNA PCR products from individual nematodes obtained from raspberry field populations and the greenhouse population. For the phylogenetic analysis, these were aligned with Wolbachia 16S rRNA sequences from four out-groups; plant-parasitic nematode $R$. similis (NCBI accession no: KF059257/EU833482) and filarial nematodes $O$. ochengi (AJ010276), B. malayi (AJ010275), and L. sigmodontis (FR827944). The outgroups were selected based on results from a previous phylogenetic analysis with $16 \mathrm{~S}$ rRNA, ftsZ, and groEL genes (Brown et al., 2016). The sequences were trimmed and $\sim 300$ bp of aligned DNA sequences were used for the phylogenetic tree generation. For phylogenetics, model testing was performed in MEGA6 to evaluate the optimal nucleotide substitution model for this data. Then, the optimal model (Kimura 2-parameter model) was utilized to generate a maximum likelihood phylogeny, evaluated with 1000 bootstrap replicates. 


\section{P. penetrans Mitochondrial DNA Sequence Analysis}

To assess whether wPpe-infected nematode lineages were genetically different from wPpe-uninfected nematodes, we analyzed the segment of the coxl mtDNA gene (a fastevolving marker) from $32 \mathrm{P}$. penetrans field populations and the greenhouse population. DNA extracted from bulk nematode samples were used for this purpose. Nematode mtDNA sequences were aligned, trimmed and then analyzed in MEGA6 as described above.

\section{Sex Ratio Determination}

Ten $P$. penetrans populations obtained from raspberry were used for sex ratio determination. We sampled 300 nematodes per population. For each population, extracted nematodes were placed on a counting slide and observed on an inverted microscope at $40 \times$ magnification. Once morphologically identified as $P$. penetrans, adult females were identified by the presence of a vulva, adult males by a bursa/spicule, and juveniles by size and lack of a developed reproductive system. Adult males, adult females, and juveniles were counted under the microscope until 100 individuals per replicate were evaluated; three replicates were counted per population (Supplementary Table S3). Percentage of males and females per replicate were calculated and the average male and female percentages per population were determined. The sex ratio of a population was determined using the average values.

\section{Statistical Analyses}

All statistical analyses were performed using R-Studio version 1.1.423. Chi-square test for homogeneity was performed (Pearson's Chi-square test), to test for any significant difference in sex ratio between the $10 \mathrm{P}$. penetrans populations obtained from raspberry; Null hypothesis $\left(\mathrm{H}_{0}\right)$ : Sex ratio is homogeneous across all $P$. penetrans populations. Chi-square tests for given probabilities (goodness of fit) were performed for each above $P$. penetrans population separately $(N=10)$, to test any deviation from the null expected ratio of 1:1 males and females in the population.

\section{RESULTS}

\section{wPpe Occurrence and Infection Prevalence in $P$. penetrans}

Among the 32 commercial field populations collected, our PCR screen of bulk nematode samples revealed the presence of wPpe in nine P. penetrans populations located in Washington, Oregon and California (Table 1). P. penetrans populations obtained from raspberry, strawberry, clover, and lily were positive for wPpe while the symbiont was not detected in $P$. penetrans populations collected from mint, cherry, apple, and pear (Table 1). When multiple field samples corresponding to a particular host plant were available, not all of them carried wPpe-infected nematodes indicating that wPpe occurrence is not specific to $P$. penetrans populations infecting specific host plants. wPpe was detected in $40 \%$ of the California P. penetrans populations, $31 \%$ of the Oregon populations, and $25 \%$ of the Washington populations. In the four infected raspberry populations, PCR screens of individual nematodes revealed variable wPpe infection levels ranging from 11 to $58 \%$ of nematodes analyzed (Table 2). Among infected raspberry $P$. penetrans populations, wPpe was observed in $12-53 \%$ of individual females compared to $8-100 \%$ of individual males (Table 2).

\section{DNA Sequence Analysis of wPpe 16S rRNA and $P$. penetrans Mitochondrial DNA}

We sequenced 54 wPpe 16S rRNA PCR products derived from both males and females from all wPpe infected raspberry populations. All amplicons had $>99 \%$ and $95 \%$ sequence identity to the homologous regions sequenced in Wolbachia from $P$. penetrans and $R$. similis, respectively (NCBI accession numbers NZ_MJMG01000007 and KF059257, respectively). A Wolbachia phylogeny based on the 16S rRNA gene region (Figure 2), using the $R$. similis-infecting Wolbachia as an outgroup placed all wPpe sequences into a single clade. Most of the individual nematodes analyzed (45/54) shared an identical wPpe sequence, while there were 9 other unique 16S rRNA sequences (Supplementary Table S4), each occurring in only one nematode. The majority of these alternate sequences contained single nucleotide polymorphisms. While most $P$. penetrans populations contained little genetic variation in wPpe 16S rRNA, one population (Custer) harbored seven different 16S rRNA sequence types (Supplementary Table S4). All 33 P. penetrans populations analyzed, including many wPpe-infected and wPpeuninfected nematodes, shared an identical coxl haplotype providing no evidence for an association between wPpe infection status and mitochondrial haplotype.

\section{Occurrence of Cardinium cPpe in $P$. penetrans Populations}

cPpe was detected in only $1 / 32$ field populations analyzed (Table 1). The cPpe-infected field population was obtained from a mint field in Washington that did not carry wPpe. The original greenhouse $P$. penetrans population was co-infected with both endosymbionts (wPpe and cPpe). PCR amplicons from cPpe matched the 16S rRNA from Cardinium in the plant-parasitic nematode H. glycines (NCBI accession number DQ314214) with $92 \%$ sequence similarity.

\section{Sex Ratio Distortion in wPpe-Infected $P$. penetrans Populations}

Male to female sex ratio in the raspberry-derived $P$. penetrans populations ranged from 1.00:0.82 to 1.00:22.00 (Table 2). Pearson's chi-square test rejected the null hypothesis that sex ratio is homogeneous across all $P$. penetrans populations obtained from raspberry $(N=10)$ and indicated that among all the $P$. penetrans populations analyzed, there was at least one population with a deviated sex ratio $\left(\chi^{2}=120, d f=9, p<0.01\right)$. The chi-square test for given probabilities (goodness of fit) supported our null hypothesis of male to female sex ratio 1:1 for 


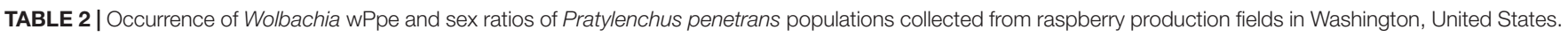

\begin{tabular}{|c|c|c|c|c|c|}
\hline Population origins & Bulk PCR & $\begin{array}{c}\text { Sex ratio } \\
\text { (M:F) }\end{array}$ & Infected males & Infected females & $\begin{array}{c}\text { wPpe } \\
\text { prevalence* (\%) }\end{array}$ \\
\hline Custer, WA & + & $1: 1.9$ & $8 / 100$ & $12 / 100$ & 10.6 \\
\hline Sumas, WA & + & $1: 3.7$ & $8 / 24$ & $44 / 100$ & 41.6 \\
\hline Everson, WA & + & $1: 5.9$ & $32 / 32$ & $51 / 100$ & 58.2 \\
\hline Greenhouse, Corvallis, OR & + & $1: 22$ & $9 / 27$ & $53 / 100$ & 52.1 \\
\hline Lynden-a, WA & - & 1:0.82 & NA & NA & NA \\
\hline Lynden-b, WA & - & $1: 1.1$ & NA & NA & NA \\
\hline Lynden-c, WA & - & $1: 1.1$ & NA & NA & NA \\
\hline Lynden-d, WA & - & $1: 1.2$ & NA & NA & NA \\
\hline Lynden -e, WA & - & $1: 1.2$ & NA & NA & NA \\
\hline Lynden-f, WA & - & $1: 1.3$ & NA & NA & NA \\
\hline
\end{tabular}

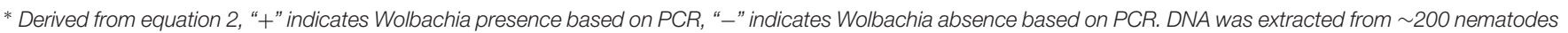
for bulk PCR, "NA," not applicable.

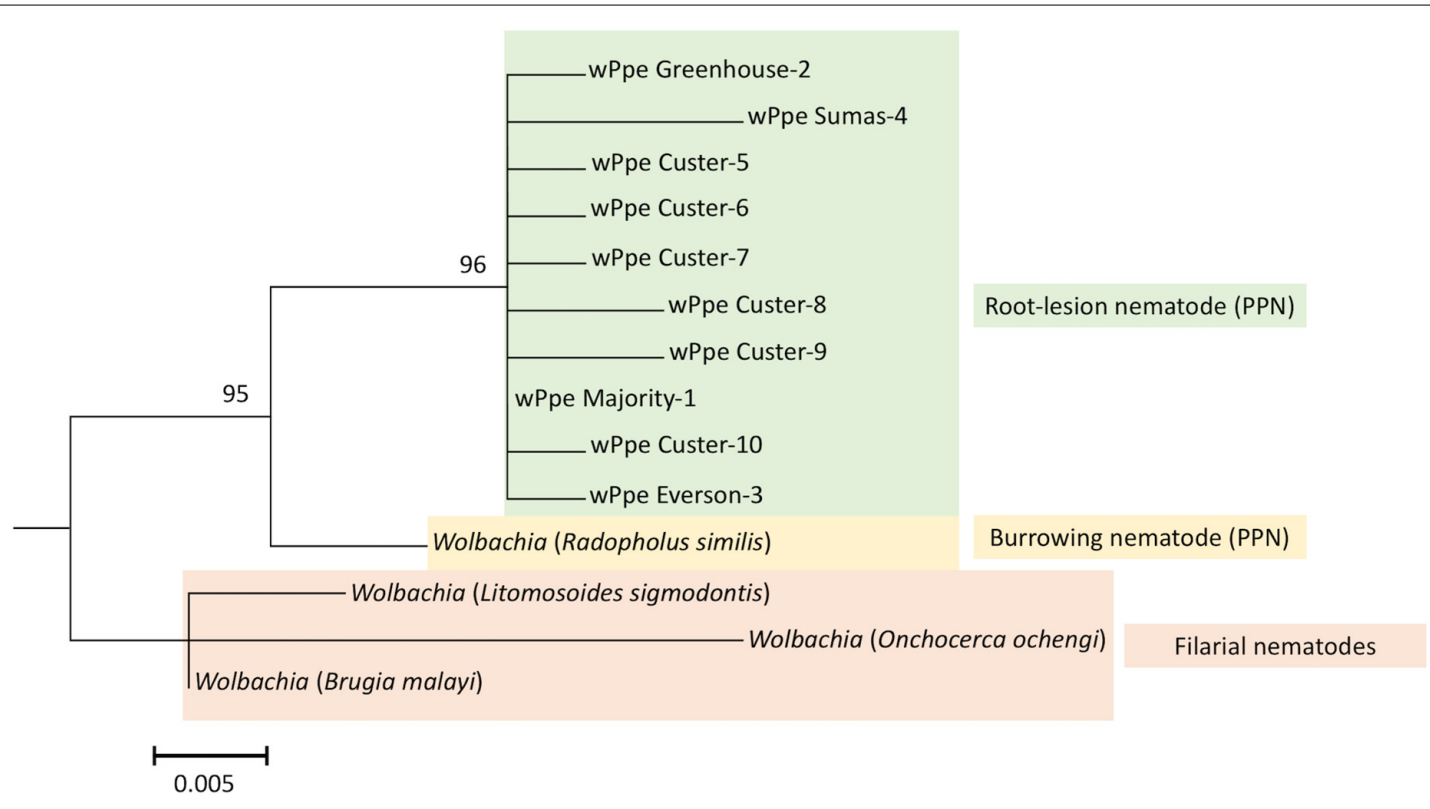

FIGURE 2 | Maximum likelihood phylogeny for Wolbachia 16S rRNA; 1000 bootstrap replications. Color indicates Wolbachia hosts; Green: Pratylenchus penetrans, Yellow: Radopholus similis, Red: Filarial nematodes. Corresponding Wolbachia hosts are indicated within brackets. Wolbachia wPpe numbers represent different $16 \mathrm{~S}$ rRNA sequence types shown in Supplementary Table S4. wPpe majority represents the 16S rRNA sequence shared by the majority of $P$. penetrans. Scale bar represents the number of substitutions per site. PPN, plant-parasitic nematode.

wPpe-uninfected populations $\left(\chi^{2}=0.6-2.2, d f=1, p>0.01\right.$, Supplementary Table S5), and rejected the null hypothesis for wPpe-infected populations $\left(\chi^{2}=6.7-76.7, d f=1, p<0.01\right.$, Supplementary Table S5), indicating that there was a sex ratio distortion (either female or male bias) when the nematodes were infected with wPpe. The percentage of female nematodes in the four wPpe-infected populations ranged from 66 to $96 \%$, while the percentage of female nematodes in the six wPpe-uninfected populations ranged from 45 to $57 \%$ (Figure 3), indicating sex ratio bias revealed by the chi-square tests was toward females. When the female percentage was graphed against Wolbachia percentage, a positive correlative trend was observed; the higher the wPpe prevalence, the higher the degree of female bias in a population (Figure 4).

\section{DISCUSSION}

Studies of natural host-symbiont populations can illuminate critical ecological and evolutionary dynamics relevant in predicting symbiont roles, and help explore the feasibility of symbiont-based biocontrol. Accordingly, this study focused on characterizing natural distribution and impact of the endosymbiont Wolbachia wPpe, a newly described, earlydiverging lineage in plant-parasitic nematodes (Brown et al., 2016; Denver et al., 2016). The first goal was to characterize the extent of wPpe infection across $P$. penetrans populations collected from commercial crop fields to assess affinity with either reproductive parasite strains or mutualist strains. This question emerged from our previous comparative genomic 


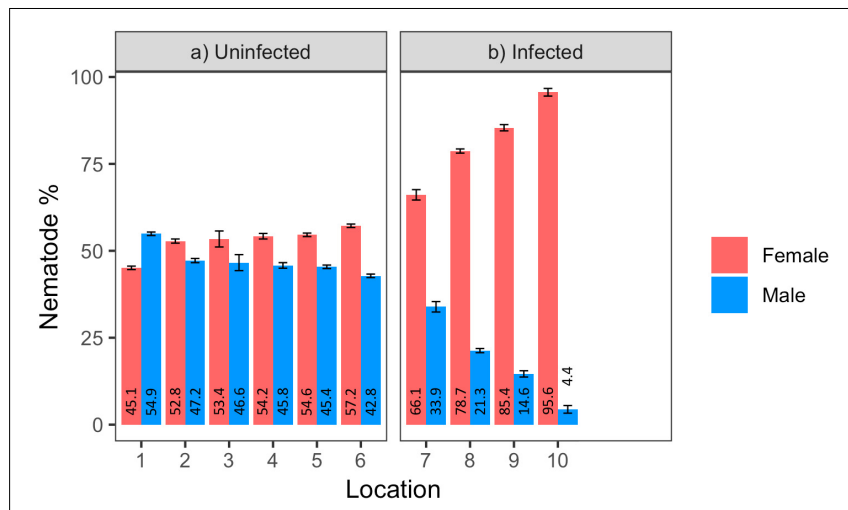

FIGURE 3 | Percentage of males and females in (a) uninfected and (b) infected Pratylenchus penetrans populations ( $N=100$ including juveniles, 3 replicates per population) from raspberry fields in Washington, United States (1-10 = locations; 1-6: Lynden, 7: Custer, 8: Sumas, 9: Everson, 10: Greenhouse). Error bars represent standard error of the mean.

analyses which could not explicitly predict the functional role of strain wPpe (Brown et al., 2016). In this study, our results clearly demonstrated that some $P$. penetrans field populations do not harbor wPpe: we found only $28 \%$ of the populations investigated were infected, a pattern that is more similar to that observed in reproductive parasite Wolbachia strains from arthropods (Weinert et al., 2015; Balvín et al., 2018). For $P$. penetrans populations found to harbor wPpe in the bulk screen, our analyses of individual nematodes revealed variable infection levels within populations. However, within-population fixation (i.e., 100\% infection levels) was never observed. This observation sharply contrasts with that in well-studied filarial nematodes in which Wolbachia is usually found in all individuals of a species (Taylor et al., 2005) due to its obligate mutualistic role. Therefore, these results suggest that wPpe and $P$. penetrans do not engage in an obligate mutualism.

The broadly variable prevalence of wPpe among $P$. penetrans populations could be explained by several causes. Vertical transmission inefficiency poses one possibility. Many Wolbachia lineages in other species do not show $100 \%$ vertical transmission efficiency from mother to offspring (Kittayapong et al., 2002; Narita et al., 2007), resulting in a proportion of eggs from infected mothers failing to inherit the symbiont. Horizontal transmission offers another potential source for dynamic prevalence data. $P$. penetrans feeds on plant roots, and there is recent evidence that Wolbachia has the capacity to transmit horizontally through plants (Li et al., 2017). In Bemisia whiteflies, for example, after infected individuals feed on leaves, Wolbachia was detected in the plant's phloem. When Wolbachia-free whiteflies subsequently fed on the infected plant leaves, they became infected and were able to vertically transmit endosymbionts to their progeny (Li et al., 2017). Finally, we investigated whether co-infection with a second reproductive manipulator, Cardinium, might affect prevalence and sex-ratio bias, but we found only one field population was infected with cPpe, with no WolbachiaCardinium co-infections, suggesting this secondary symbiont did not contribute to the patterns observed here.

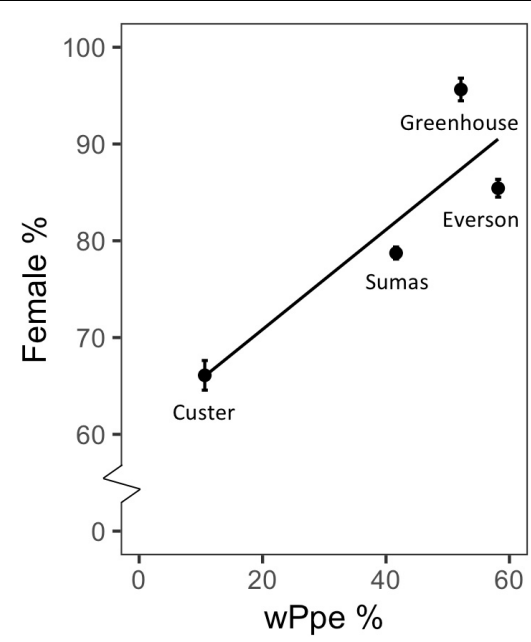

FIGURE 4 | Relationship between sex ratio (percentage females) and percentage of individuals infected with Wolbachia wPpe of Pratylenchus penetrans populations from raspberry fields in Washington, United States.

The second goal of this study was to characterize $P$. penetrans sex ratios across field populations to evaluate whether wPpe has the population characteristics of a sex ratio distorter. In most sexually reproducing animals, the sex ratio is maintained at, or close to, 1:1 by frequency-dependent selection (Fisher, 1930). We observed that sex ratios of the wPpe-uninfected $P$. penetrans populations were not significantly different from 1:1 while infected populations were female biased. Furthermore, the female bias in wPpe-infected $P$. penetrans populations was highly significant $(p<0.01)$ and there was a positive correlation between the degree of female bias and the prevalence of Wolbachia. This observation was surprising considering past studies have shown that $P$. penetrans is strictly sexual and typically displays a 1:1 sex ratio (Roman and Triantaphyllou, 1969). Sex determination in arthropods and nematodes are often genetically regulated where chromosomal content determines the sex of an individual. However, studies have revealed that in arthropods, sex determination can be manipulated by bacterial endosymbionts, such as Wolbachia (Cordaux et al., 2011; Kageyama et al., 2012). For example, Wolbachia was associated with extreme female bias in Cordylochernes pseudoscorpions; antibiotic treatment cured females of the Wolbachia infection and restored offspring sex ratios to 1:1 (Zeh et al., 2005). In Oniscus woodlice and Eurema butterflies, females infected with Wolbachia produced a higher proportion of females in their broods as compared to uninfected females (Rigaud et al., 1999; Kern et al., 2015).

Our observation of female bias in the wPpe-infected populations could be due to one or more Wolbachia-induced reproductive abnormalities such as male killing, feminization of biological males, and parthenogenesis. While there is currently no experimental evidence for male-killing, feminization, or parthenogenesis in wPpe, our data showed a variable prevalence of wPpe in males (8-100\%) for the female-biased populations. While infection in males has been reported in arthropods and 
filarial nematodes (Werren et al., 2008; Slatko et al., 2010), high wPpe prevalence in males would be unexpected under a malekilling phenotype unless the male-killing phenotype occurred late in the development. Similarly, high wPpe prevalence in males would be unexpected in parthenogenesis unless parthenogenesis is weak or incomplete. Incomplete feminization, on the other hand, might be consistent with this data, although males still would not be expected to have higher infection rates than females under this scenario. Our observations might also be influenced by unknown wPpe effects on male nematodes, such as male development rates and dispersion phenotypes. To fully understand the cause of Wolbachia-associated sexratio bias will require further experimentation using antibiotic treatment and life history comparisons in the $P$. penetranswPpe system.

Previously, genomic and tissue distribution analyses of wPpe showed some patterns consistent with this symbiont having the potential to have reproductive manipulation effects (Brown et al., 2016). For example, core and pangenome analyses presented some gene repertoire similarities between wPpe and reproductive manipulators. Further, fluorescent insitu hybridization experiments revealed a dispersed tissue distribution of wPpe (localized throughout the tissues of $P$. penetrans including the head, pharynx intestine, and ovaries), resembling the tissue distribution patterns found in other Wolbachia strains known to be reproductive manipulators (Brown et al., 2016). Conversely, most obligate mutualist Wolbachia strains are concentrated in specialized tissues such as the lateral cords (Slatko et al., 2010; Fischer et al., 2011). The genome of wPpe showed no evidence for the presence of CI gene homologs or WO phage genes (Brown et al., 2016), which have recently been implicated as causative of CI (Beckmann and Fallon, 2014; Le Page et al., 2017; Bordenstein et al., 2018). However, wPpe's evolutionary position as an earlydiverging lineage raises the possibility that it might induce CI or similar phenotypes through alternative and unknown genetic mechanisms. In filarial nematodes, Wolbachia does not exhibit selfish behaviors such as reproductive manipulation. By contrast, our findings suggest that wPe might function as a reproductive parasite, similar to Wolbachia found in arthropods. Indeed, wPpe has a larger genome than that of filarial nematode Wolbachia strains (Brown et al., 2016), supporting the idea that it may harbor more capacity to manipulate hosts.

Another Wolbachia strain related to wPpe infects the plantparasitic nematode $R$. similis, but its symbiotic impacts on the host remain unclear. The report describing Wolbachia in $R$. similis suggests an essential function of the endosymbiont based on its prevalence at 100\% (Haegeman et al., 2009). However, this was based upon a small sample size, 28 females and 29 males, cultured on carrot discs derived from a population in Uganda. In addition, Wolbachia was reported to be present in another $R$. similis population obtained from Indonesia and in a closely related species $R$. arabocoffeae (Haegeman et al., 2009). Additional surveys of $R$. similis field populations might illuminate whether this strain also has variable prevalence similar to that reported here for $P$. penetrans. So far, there is limited information on the presence or absence of Wolbachia among plant-parasitic nematode species, with no recent systematic surveys published to date (Brown, 2018). A genome skimming strategy applied to six plant-parasitic nematode species provided no evidence for Wolbachia in five of the screened species (Denver et al., 2016) supporting the non-obligatory function of Wolbachia in the plant-parasitic nematodes.

\section{CONCLUSION}

In conclusion, this study demonstrated that $P$. penetrans carrying wPpe occur across a wide geographical range and in a variety of host plants at variable wPpe prevalence that never attained $100 \%$. Therefore, unlike Wolbachia symbioses in filarial nematodes, wPpe does not appear to be required and is not an obligate mutualist of $P$. penetrans. The observed correlation between female-biased sex ratio and Wolbachia prevalence, suggests that wPpe might act as a reproductive manipulator, but more work is required to confirm this. Future studies should investigate controlled genetic crosses and antibiotic elimination of Wolbachia. Such experimental strategies would be, challenging for $P$. penetrans, an obligatory endoparasitic and understudied nematode species. Although it has been difficult to study fitness directly in these obligate migratory endo-parasites of plant roots, it has become possible to culture P. penetrans on excised roots, which might open new experimental opportunities for this emerging symbiosis system.

\section{AUTHOR CONTRIBUTIONS}

SW, DD, AB, and IZ designed the experiments. IZ, DD, DH, and AP collected the root and soil samples. SW, DH, and JK performed the experiments. SW analyzed the data and wrote the manuscript with input from all the co-authors. All the authors approved the final version of the manuscript.

\section{FUNDING}

This work was supported by the United States Department of Agriculture grant SCA 58-5358-3-026.

\section{ACKNOWLEDGMENTS}

We would like to thank Sara Trainer and Jessica Nixon at the Oregon State University Center for Genome Research and Biocomputing for the DNA sequencing assistance. We also thank all members of the Denver lab for their valuable inputs in discussions relevant to this study.

\section{SUPPLEMENTARY MATERIAL}

The Supplementary Material for this article can be found online at: https://www.frontiersin.org/articles/10.3389/fmicb.2019. 00964/full\#supplementary-material 


\section{REFERENCES}

Arakaki, N., Miyoshi, T., and Noda, H. (2001). Wolbachia-mediated parthenogenesis in the predatory thrips Franklinothrips vespiformis (Thysanoptera: Insecta). Proc. Biol. Sci. 268, 1011-1016. doi: 10.1098/ rspb. 2001.1628

Atibalentja, N., and Noel, G. R. (2008). Bacterial endosymbionts of plant-parasitic nematodes. Symbiosis 46, 87-93.

Balvín, O., Roth, S., Talbot, B., and Reinhardt, K. (2018). Co-speciation in bedbug Wolbachia parallel the pattern in nematode hosts. Sci. Rep. 8, 1-9. doi: 10.1038/ s41598-018-25545-y

Bandi, C., McCall, J. W., Genchi, C., Corona, S., Venco, L., and Sacchi, L. (1999). Effects of tetracycline on the filarial worms Brugia pahangi and Dirofilaria immitis and their bacterial endosymbionts Wolbachia. Int. J. Parasitol. 29, 357-364. doi: 10.1016/S0020-7519(98)00200-8

Beckmann, J. F., and Fallon, A. M. (2014). Spermathecae: implications for cytoplasmic incompatibility. Insect Biochem. Mol. Biol. 43, 867-878. doi: 10. 1016/j.ibmb.2013.07.002

Bird, D. M. K., and Kaloshian, I. (2003). Are roots special? Nematodes have their say. Physiol. Mol. Plant Pathol. 62, 115-123. doi: 10.1016/S0885-5765(03) 00045-6

Bordenstein, S. R., Layton, E. M., Zhou, H., Shropshire, J. D., and On, J. (2018). One prophage WO gene rescues cytoplasmic incompatibility in Drosophila melanogaster. Proc. Natl. Acad. Sci. U.S.A. 115, 4987-4991. doi: 10.1073/pnas. 1800650115

Bourtzis, K., Nirgianaki, A., Markakis, G., and Savakis, C. (1996). Wolbachia infection and cytoplasmic incompatibility in Drosophila species. Genetics 144, 1063-1073. doi: 10.1186/1471-2105-15-293

Brelsfoard, C. L., and Dobson, S. L. (2009). Wolbachia-based strategies to control insect pests and disease vectors. AsPac J. Moecular Biol. Biotechnol. 17, 55-63.

Brown, A. M. V. (2018). Endosymbionts of plant-parasitic nematodes. Annu. Rev. Phytopathol. 56, 225-242. doi: 10.1146/annurev-phyto-080417-045824

Brown, A. M. V., Wasala, S. K., Howe, D. K., Peetz, A. B., Zasada, I. A., and Denver, D. R. (2016). Genomic evidence for plant-parasitic nematodes as the earliest Wolbachia hosts. Sci. Rep. 6:34955. doi: 10.1038/srep34955

Brown, A. M. V., Wasala, S. K., Howe, D. K., Peetz, A. B., Zasada, I. A., and Denver, D. R. (2018). Comparative genomics of Wolbachia-Cardinium dual endosymbiosis in a plant-parasitic nematode. Front. Microbiol. 9:2482. doi: 10.3389/fmicb.2018.02482

Cordaux, R., Bouchon, D., and Grève, P. (2011). The impact of endosymbionts on the evolution of host sex-determination mechanisms. Trends Genet. 27, 332-341. doi: 10.1016/j.tig.2011.05.002

Denver, D. R., Brown, A. M. V., Howe, D. K., Peetz, A. B., and Zasada, I. A. (2016). Genome skimming: a rapid approach to gaining diverse biological insights into multicellular pathogens. PLoS Pathog. 12:e1005713. doi: 10.1371/journal.ppat. 1005713

Dyson, E. A., and Hurst, G. D. D. (2004). Persistence of an extreme sex-ratio bias in a natural population. Proc. Natl. Acad. Sci. U.S.A. 101, 6520-6523. doi: $10.1073 /$ pnas.0304068101

Endo, B. Y. (1979). The ultrastructure and distribution of an intracellular bacterium-like microorganism in tissues of larvae of the soybean cyst nematode, Heterodera glycines. J. Ultrasructure Res. 67, 1-4. doi: 10.1016/S0022-5320(79) 80012-X

Fialho, R. F., and Stevens, L. (2000). Male-killing Wolbachia in a flour beetle. Proc. Biol. Sci. 267, 1469-1473. doi: 10.1098/rspb.2000.1166

Fischer, K., Beatty, W. L., Jiang, D., Weil, G. J., and Fischer, P. U. (2011). Tissue and stage-specific distribution of Wolbachia in Brugia malayi. PLoS Negl. Trop. Dis. 5:e1174. doi: 10.1371/journal.pntd.0001174

Fisher, R. A. (1930). The genetical theory of natural selection. Genetics 154:272. doi: $10.1038 / 158453 \mathrm{a} 0$

Flores, H. A., and O'Neill, S. L. (2018). Controlling vector-borne diseases by releasing modified mosquitoes. Nat. Rev. Microbiol. 16, 508-518. doi: 10.1038/ s41579-018-0025-0

Fransolet, D., Roberty, S., and Plumier, J. C. (2012). Establishment of endosymbiosis: the case of cnidarians and Symbiodinium. J. Exp. Mar. Bio. Ecol. 42, 1-7. doi: 10.1016/j.jembe.2012.03.015

Gigot, J., Walters, T. W., and Zasada, I. A. (2013). Impact and occurrence of Phytophthora rubi and Pratylenchus penetrans in commercial red raspberry
(Rubus ideaus) fields in Northwestern Washington. Int. J. Fruit Sci. 13, 357-372. doi: $10.1080 / 15538362.2013 .748373$

Goffredi, S. K. (2010). Indigenous ectosymbiotic bacteria associated with diverse hydrothermal vent invertebrates. Environ. Microbiol. Rep. 2, 479-488. doi: 10. $1111 /$ j.1758-2229.2010.00136.x

Haegeman, A., Vanholme, B., Jacob, J., Vandekerckhove, T. T. M., Claeys, M., Borgonie, G., et al. (2009). An endosymbiotic bacterium in a plant-parasitic nematode: member of a new Wolbachia supergroup. Int. J. Parasitol. 39, 1045-1054. doi: 10.1016/j.ijpara.2009.01.006

Hertig, M. (1936). The Rickettsia, Wolbachia pipientis (gen. et sp.n.) and associated inclusions of the mosquito, Culex pipiens. Parasitology 28:453. doi: 10.1017/ S0031182000022666

Hoerauf, A., Nissen-Pähle, K., Schmetz, C., Henkle-Dührsen, K., Blaxter, M. L., Büttner, D. W., et al. (1999). Tetracycline therapy targets intracellular bacteria in the filarial nematode Litomosoides sigmodontis and results in filarial infertility. J. Clin. Invest. 103, 11-18. doi: 10.1172/JCI4768

Hurst, G. D., Johnson, A. P., Schulenburg, J. H., and Fuyama, Y. (2000). Malekilling Wolbachia in Drosophila: a temperature-sensitive trait with a threshold bacterial density. Genetics 156, 699-709.

Jiggins, F. M., Hurst, G. D. D., Dolman, C. E., and Majerus, M. E. N. (2000). Highprevalence male-killing Wolbachia in the butterfly Acraea encedana. J. Evol. Biol. 13, 495-501. doi: 10.1046/j.1420-9101.2000.00180.x

Jones, J. T., Haegeman, A., Danchin, E. G. J., Gaur, H. S., Helder, J., Jones, M. G. K., et al. (2013). Top 10 plant-parasitic nematodes in molecular plant pathology. Mol. Plant Pathol. 14, 946-961. doi: 10.1111/mpp.12057

Kageyama, D., Narita, S., and Watanabe, M. (2012). Insect sex determination manipulated by their endosymbionts: incidences, mechanisms and implications. Insects 3, 161-199. doi: 10.3390/insects3 010161

Kageyama, D., Nishimura, G., Hoshizaki, S., and Ishikawa, Y. (2002). Feminizing Wolbachia in an insect, Ostrinia furnacalis (Lepidoptera: Crambidae). Heredity 88, 444-449. doi: 10.1038/sj/hdy/6800077

Kern, P., Cook, J. M., Kageyama, D., and Riegler, M. (2015). Double trouble: combined action of meiotic drive and Wolbachia feminization in Eurema butterflies. Biol. Lett. 11:20150095. doi: 10.1098/rsbl.2015.0095

Kittayapong, P., Baisley, K. J., Sharpe, R. G., Baimai, V., and O'Neill, S. L. (2002). Maternal transmission efficiency of Wolbachia superinfections in Aedes albopictus populations in Thailand. Am. J. Trop. Med. Hyg. 66, 103-107. doi: 10.4269/ajtmh.2002.66.103

Kumar, S., Nei, M., Dudley, J., and Tamura, K. (2008). MEGA: a biologistcentric software for evolutionary analysis of DNA and protein sequences. Brief. Bioinform. 9, 299-306. doi: 10.1093/bib/bbn017

Lamelas, A., Gosalbes, M. J., Manzano-Marín, A., Peretó, J., Moya, A., and Latorre, A. (2011). Serratia symbiotica from the aphid Cinara cedri: a missing link from facultative to obligate insect endosymbiont. PLoS Genet. 7:e1002357. doi: 10.1371/journal.pgen.1002357

Langworthy, N. G., Renz, A., Mackenstedt, U., Henkle-Dührsen, K., de Bronsvoort, M. B., Tanya, V. N., et al. (2000). Macrofilaricidal activity of tetracycline against the filarial nematode Onchocerca ochengi: elimination of Wolbachia precedes worm death and suggests a dependent relationship. Proc. Biol. Sci. 267, 1063-1069. doi: 10.1098/rspb.2000.1110

Le Page, D. P., Metcalf, J. A., Bordenstein, S. R., On, J., Perlmutter, J. I., Shropshire, J. D., et al. (2017). Prophage WO genes recapitulate and enhance Wolbachia-induced cytoplasmic incompatibility. Nature 543, 243-247. doi: 10. 1038/nature21391

Li, S. J., Ahmed, M. Z., Lv, N., Shi, P. Q., Wang, X. M., Huang, J. L., et al. (2017). Plantmediated horizontal transmission of Wolbachia between whiteflies. ISME J. 11, 1019-1028. doi: 10.1038/ismej.2016.164

Moya, A., Peretó, J., Gil, R., and Latorre, A. (2008). Learning how to live together: genomic insights into prokaryote-animal symbioses. Nat. Rev. Genet. 9, 218 229. doi: $10.1038 / \mathrm{nrg} 2319$

Narita, S., Nomura, M., and Kageyama, D. (2007). Naturally occurring single and double infection with Wolbachia strains in the butterfly Eurema hecabe: transmission efficiencies and population density dynamics of each Wolbachia strain. FEMS Microbiol. Ecol. 61, 235-245. doi: 10.1111/j.1574-6941.2007. 00333.x

Negri, I., Pellecchia, M., Mazzoglio, P. J., Patetta, A., and Alma, A. (2006). Feminizing Wolbachia in Zyginidia pullula (Insecta, Hemiptera), a leafhopper 
with an XX/X0 sex-determination system. Proc. Biol. Sci. 273, 2409-2416. doi: 10.1098/rspb.2006.3592

Noel, G. R., and Atibalentja, N. (2006). "Candidatus Paenicardinium endonii" an endosymbiont of the plant-parasitic nematode Heterodera glycines (Nemata: Tylenchida), affiliated to the phylum Bacteroidetes. Int. J. Syst. Evol. Microbiol. 56, 1697-1702. doi: 10.1099/ijs.0.64234-0

Rigaud, T., Moreau, J., and Juchault, P. (1999). Wolbachia infection in the terrestrial isopod Oniscus asellus: sex ratio distortion and effect on fecundity. Heredity 83, 469-475. doi: 10.1038/sj.hdy.6885990

Roman, J., and Triantaphyllou, A. C. (1969). Gametogenesis and reproduction of seven species of Pratylenchus. J. Nematol. 1, 357-362.

Shepherd, A. M., Clark, S. A., and Kempton, A. (1973). An intracellular microorganism associated with tissues of Heterodera Spp. Nematologica 19, 31-34. doi: 10.1163/187529273X00060

Sicard, M., Bouchon, D., Ceyrac, L., Raimond, R., Thierry, M., Le Clec'h, W., et al. (2014). Bidirectional cytoplasmic incompatibility caused by Wolbachia in the terrestrial isopod Porcellio dilatatus. J. Invertebr. Pathol. 121, 28-36. doi: 10.1016/j.jip.2014.06.007

Slatko, B. E., Luck, A. N., Dobson, S. L., and Foster, J. M. (2014). Wolbachia endosymbionts and human disease control. Mol. Biochem. Parasitol. 195, 88-95. doi: 10.1016/j.molbiopara.2014.07.004

Slatko, B. E., Taylor, M. J., and Foster, J. M. (2010). The Wolbachia endosymbiont as an anti-filarial nematode target. Symbiosis 51, 55-65. doi: 10.1007/s13199-0100067-1

Stouthamer, R., Luck, R. F., and Hamilton, W. D. (1990). Antibiotics cause parthenogenetic Trichogramma (Hymenoptera/Trichogrammatidae) to revert to sex. Proc. Natl. Acad. Sci. U.S.A. 87, 2424-2427. doi: 10.1073/pnas. 87.7.2424

Tarte, R., and Mai, W. F. (1976). Sex expression and tail morphology of female progenies of smooth-tail and crenate-tail females of Pratylenchus penetrans. J. Nematol. 8, 196-199.

Taylor, M. J., Bandi, C., and Hoerauf, A. (2005). Wolbachia bacterial endosymbionts of filarial nematodes. Adv. Parasitol. 60, 245-284. doi: 10.1016/ S0065-308X(05)60004-8

Turner, J. D., Sharma, R., Al Jayoussi, G., Tyrer, H. E., Gamble, J., Hayward, L., et al. (2017). Albendazole and antibiotics synergize to deliver shortcourse anti- Wolbachia curative treatments in preclinical models of filariasis. Proc. Natl. Acad. Sci. U.S.A. 114, E9712-E9721. doi: 10.1073/pnas.1710 845114

Vandekerckhove, T. T. M., Watteyne, S., Bonne, W., Vanacker, D., Devaere, S., Rumes, B., et al. (2003). Evolutionary trends in feminization and intersexuality in woodlice (Crustacea, Isopoda) infected with Wolbachia pipientis (alphaProteobacteria). Belgian J. Zool. 133, 61-69.

Walsh, J. A., Shepherd, A. M., and Lee, D. L. (1983a). The distribution and effect of intracellular rickettsia-like micro-organisms infecting second-stage juveniles of the Potato cyst- nematode Globodera rostochiensis. J. Zool. 199, 395-419. doi: 10.1111/j.1469-7998.1983.tb02104.x

Walsh, J. A., Walsh, J. A., Lee, D. L., and Shepherd, A. M. (1983b). The distribution and effect of intracellular Rickettsia-like micro-organisms infecting adult males of the potato cyst-nematode Globodera Rostochiensis. Nematologica 29, 227-239. doi: 10.1163/187529283X00474

Weeks, A. R., and Breeuwer, J. A. J. (2001). Wolbachia-induced parthenogenesis in a genus of phytophagous mites. Proc R Soc L. B 268, 2245-2251. doi: 10.1098/ rspb.2001.1797

Weinert, L. A., Araujo-Jnr, E. V., Ahmed, M. Z., and Welch, J. J. (2015). The incidence of bacterial endosymbionts in terrestrial arthropods. Proc. R. Soc. B Biol. Sci. 282, 3-8. doi: 10.1098/rspb.2015.0249

Werren, J. H., Baldo, L., and Clark, M. E. (2008). Wolbachia: master manipulators of invertebrate biology. Nat. Rev Microbiol 6, 741-751. doi: 10.1038/ nrmicro1969

Wolfgang, A., Markus, R., Dimitrios, N. A., and Christian, S. (2009). Evidence for low-titre infections in insect symbiosis: Wolbachia in the bark beetle Pityogenes chalcographus (Coleoptera, Scolytinae). Environ. Microbiol. 11, 1923-1933. doi: 10.1111/j.1462-2920.2009.01914.x

Xie, R. R., Sun, J. T., Xue, X. F., and Hong, X. Y. (2016). Cytoplasmic incompatibility and fitness benefits in the two-spotted spider mite Tetranychus urticae (red form) doubly infected with Wolbachia and Cardinium. Syst. Appl. Acarol. 21, 1161-1173. doi: 10.11158/saa.21.9.1

Zabalou, S., Riegler, M., Theodorakopoulou, M., Stauffer, C., Savakis, C., and Bourtzis, K. (2004). Wolbachia-induced cytoplasmic incompatibility as a means for insect pest population control. Proc. Natl. Acad. Sci. U.S.A. 101, 1504215045. doi: 10.1073/pnas.0403853101

Zeh, D. W., Zeh, J. A., and Bonilla, M. M. (2005). Wolbachia, sex ratio bias and apparent male killing in the harlequin beetle riding pseudoscorpion. Heredity 95, 41-49. doi: 10.1038/sj.hdy.6800666

Zug, R., and Hammerstein, P. (2014). Bad guys turned nice? A critical assessment of Wolbachia mutualisms in arthropod hosts. Biol. Rev. 90, 89-111. doi: 10.1111/ brv.12098

Conflict of Interest Statement: The authors declare that the research was conducted in the absence of any commercial or financial relationships that could be construed as a potential conflict of interest.

Copyright @ 2019 Wasala, Brown, Kang, Howe, Peetz, Zasada and Denver. This is an open-access article distributed under the terms of the Creative Commons Attribution License (CC BY). The use, distribution or reproduction in other forums is permitted, provided the original author(s) and the copyright owner(s) are credited and that the original publication in this journal is cited, in accordance with accepted academic practice. No use, distribution or reproduction is permitted which does not comply with these terms. 